\title{
What will be the new normal for the dental industry?
}

\author{
Edmund Proffitt ${ }^{1}$
}

\section{Key points}

Reducing and mitigating the infection risks of dental aerosol generating procedures (AGPS)

will have to underpin any return to the more widespread provision of treatment.
The dental industry will work closely with the dental profession to explore, adopt and provide the necessary approaches, products, technologies and treatments to facilitate the resumption of wider dental activities.
Inevitably, the 'new' normal will mean some changes to the structure and delivery of NHS and private dentistry for some period to come.

\begin{abstract}
The COVID-19 situation has seen the cessation of all non-urgent dental care in the UK. Regular practice activity has come to a virtual standstill and the dental industry has seen a very significant reduction in its provision of products and services. There are differing government financial mitigations in place across dental practices for NHS and, to an extent private, activity, as well as for the dental industry. Dentistry is generally classified in the very high-risk category of aerosol production through many aerosol generating procedures (AGPs). To facilitate any move towards the resumption of widespread dental treatment, possibly without mass vaccination or the widespread use of antibody testing, the aerosol issue will need to be addressed and solved. From a dental industry perspective, equipment and product manufacturers and suppliers are building upon, and further developing, the industry's extremely close relationship and partnership with the dental profession, in order to develop and implement new ways of thinking and new approaches, products and techniques to protect patients and clinicians, focusing on a return to the more widespread provision of dentistry.
\end{abstract}

As a nation, we are now many weeks into coronavirus lockdown and it seems highly likely that this will continue in one form or another for some months. Under government advice, all routine, non-urgent dental care including orthodontics has been ceased and deferred until advised. Emergency treatment is being undertaken at dedicated urgent care centres established within the NHS England jurisdiction, with similar arrangements in the devolved administrations. From a dental industry perspective, the sales of consumables, materials and equipment have effectively ground to a halt and are now at a tiny fraction of what they were only a month or so ago.

In response to the unprecedented situation that the UK, and the rest of the world, is in, most UK dental companies are making use of

${ }^{1}$ Chief Executive, British Dental Industry Association, Mineral Lane, Chesham, Buckinghamshire, HP5 1NL, UK. ${ }^{*}$ Correspondence to: Edmund Proffitt

Email address: EdmundProffitt@bdia.org.uk

Accepted 20 April 2020

https://doi.org/10.1038/s41415-020-1583-x the current support measures made available by the government. British Dental Industry Association (BDIA) members encompass many different areas of trading, embracing equipment, consumables, manufacturing, distribution, support services, engineering, education, advertising, marketing and publishing. Very much like dental practices, there is no 'one size fits all' when it comes to weathering the storm.

There are differing government mitigations in place across dental practices, to a greater or lesser degree, for NHS practices and the private sector, and those inbetween. Some of these are the same non-sector specific assistance that is being taken up by the industry. Many dental companies have decided to furlough a significant number of staff, many have nearly all their staff working from home where they can, some are looking at government-backed loans, and companies will benefit in the short term from delayed VAT payments. Many are also suffering delays and postponements in customer payments.

At the moment, no one can be sure when we can return to any sort of 'normality' in our professional and home lives. Right across the sector, whether a practice or supplier, we all sincerely hope that every organisation can ride out these unprecedented times and rebuild business once circumstances change, but no doubt there will be some business casualties. However, patient treatment and business will come back, but it will inevitably not be the same and will be conducted in some new and different ways by both dental suppliers and dentists.

From an industry perspective, moving forwards, many BDIA members will have a critical and fundamental role to play in getting dentistry back in action, with an opportunity to help and shape the way that the profession once again begins to provide more dental care going forwards.

In this short paper, I want to have a quick look at a few factors that will prove crucial in moving towards the new 'normal':

1. Aerosol generating procedures (AGPs) the aerosol issue

2. When?

3. New ways of safe treatment - getting back on our feet

4. New ways of doing business. 


\section{AGPs - the aerosol issue}

Government advice as of 12 April on procedures stated: 'The highest risk of transmission of respiratory viruses is during AGPs of the respiratory tract, and use of enhanced respiratory protective equipment is indicated for health and social care workers performing or assisting in such procedures...A long-sleeved disposable fluid repellent gown (covering the arms and body) or disposable fluid repellent coveralls, a filtering face piece class 3 (FFP3) respirator, a full-face shield or visor and gloves are recommended during AGPs on possible and confirmed cases, regardless of the clinical setting.' This was further compounded by NHS England's COVID-19 standard operating procedure guidance and an update from the Chief Dental Officer addressing urgent dental care provision issued on 15 April.

Dentistry is generally classified in the very high-risk category of occupations involved with aerosol production. This is not helped by coronavirus particles ranging from 60 to 140 nanometres ( 0.06 to 0.14 micrometres), with an average of 0.125 micron, which makes them a significant aerosol threat. The majority of everyday dental procedures that use low- or high-speed handpieces, electrosurgery units, ultrasonic scalers, air polishers, prophy angles, hand instruments, air/water syringes or lasers can create bioaerosols, with everyday routine equipment like ultrasonic scalers and highspeed handpieces producing more airborne contamination than any other devices. As we have seen, the significant aerosol risk attributed to the majority of routine dental treatments has led to the suspension of general dentistry and to emergency treatment being undertaken in dedicated urgent care centres using 'full' personal protective equipment (PPE) as outlined in official guidance.

To move back to the provision of regular dental treatment without mass vaccination or the widespread use of antibody testing and COVID-19 passports, if such a thing was practical, the aerosol issue will need to be addressed and solved.

In the longer term, when we are past the infection peak and moving into possibly less strict lockdown and containment regimes, there will be a time when the resumption of more widespread dental treatment can be considered. However, measures such as disease and antibody testing for clinicians and patients would be key to this, and this could take considerable time. The availability of a vaccine will massively accelerate the return to wider normality.

As we look towards an initial broader resumption of treatments, the profession will be looking to the industry to help solve issues, particularly aerosol creation and mitigation, and that is where members can be in a position to possibly supply equipment to help address the massive 'aerosol challenge'. We look at possible options later on in this paper.

\section{When?}

There are many views on how and when lockdown could be lifted or reduced and how we can edge back to a more normal position. There will be many learnings from what is now happening in other comparable countries such as Italy and Spain, which we are all following very closely. In effect, we are all part of a great big COVID-19 experiment.

At the moment, the UK government is keeping its cards close to its chest regarding any possible lockdown 'easing' strategies and, quite rightly, playing the science card. However, looking at what is happening around the world and where we are with testing and social distancing mechanisms, it is not inconceivable that some more 'regular' dental services in the UK are unlikely to resume before Q3 of 2020 at the very earliest, if the aerosol issue can be dealt with, and will not return to pre-COVID-19 levels this year, or indeed into Q1+ of next year.

The reasons for this are primarily that strategies of suppression are likely to be successful in reducing the virus transmission over shorter periods, which we are seeing global evidence of now, leading to opportunities to relax these suppressions. However, it is thought that this could lead to another (reduced) peak in infections (possibly October 2020 in the UK), at which time some restrictions are likely to be reactivated. As things are once again brought under control with a 'second lockdown', more measures would again be relaxed, leading in turn to another, smaller, peak in infections (possibly around the end of the year). Depending on the availability of a vaccine and the level and sophistication of any testing regimes, we could lurch between these scenarios for the next 3-18 months.

Until the accurate identification of carriers of the virus is possible, and/or the extensive use of a vaccine, it is not clear to see how there could be a complete resumption of 'normality' in dental provision. However, underlying this, new methods, practices, treatments and technologies could contribute significantly to a return to more regular and widespread treatment provision.

\section{New ways of safe treatment - getting back on our feet}

Going forwards, we have seen that the provision of accurate and cost-effective disease testing and a vaccine will be key to the resumption of widespread treatment, and this will take time. However, other mitigating activities could contribute significantly to the recovery while the other pharmaceutical and IVD areas of action are being developed and implemented.

In terms of physical risk mitigation, it is generally understood that the risks of dental aerosols can be reduced with the use of highvelocity air evacuation, wearing the correct PPE, using advanced filtration, purification and decontamination systems, and, where appropriate, pre-procedural antimicrobial mouth rinses, as well as waterline disinfection discipline. Cleaning and disinfection regimes in the surgery and for equipment will also be critical. During treatment, the sites showing the highest microbiological contamination due to aerosol are visors and masks, the unit lamp, surfaces close to spittoons and mobile instrument material tables. Some other suggested mitigations include dental practices installing negative pressure rooms or airborne infection isolation rooms in which procedures generating aerosol can be performed.

These mitigations are not quick and simple undertakings, either individually or in combination, and would add a significant additional level of cost and complexity in the surgery, where indeed they could be practically implemented and where the investment is available. 'Physical' mitigations will make treatments more time-consuming and expensive, and will impact massively on the number of daily procedures undertaken and on all surgery and practice services and facilities. However, they would also be a way of providing some wider access to treatment and providing reassurance to clinicians and patients alike.

We can see how all this would impact greatly on the current 'model' of NHS dentistry, along with private provision, and there would certainly need to be some difficult decisions 
made on what the shorter term, post-urgent care centre provision of dental care would look like, how it would be rolled out and how it would be funded.

In the shorter term recovery period (up to the next $12+$ months), it is apparent that significant efforts to reduce the risk to staff and patients from aerosol exposure will be necessary. This will require close and complex cooperation between the dental industry and profession, in order to look at ways of extending risk mitigation by adopting new and more stringent protective and disinfection procedures and products, including PPE, and possibly air filtration, extraction and pressure management technologies.

Supporting the approaches to enhanced infection control, there are also significant opportunities for the adoption of new products, technologies and techniques for treatments. In the drive to reduce aerosol generation, there are opportunities for developing and expanding minimally invasive activities and a different approach to treatments and products, such as the use of silver diamine fluoride, to name one. However, there will remain challenges for a number of activities, including endodontic and orthodontic treatments and implantology, as well as some hygiene and therapeutic activity.

At this time of great uncertainty, the dental industry has a critical role to play alongside clinicians in the recovery sequence. There is an opportunity going forwards to build upon and further develop the industry's extremely close relationship and partnership with the dental profession, and to develop and implement new ways of thinking and new approaches, products and techniques to protect patients and clinicians, and allow some return to normal provision of dentistry.

\section{New ways of doing business}

Apart from the challenges of restarting more general dental provision, this period has provided organisations with an enforced opportunity to review how they structure and conduct their business going forwards. The current pandemic will have, as one of its many legacies, a profound impact on how business will be done in future. Here, we look at a number of business scenarios and practices common across the dental sector.

\section{The office environment}

Widespread working from home and a reduction in travel will impact on the way organisations look at cost-effectively using and staffing offices and facilities in future. Much smaller premises may be appropriate for a number of organisations.

\section{Business travel}

Again, widespread changes will provide useful data on the benefits/cost-effectiveness of those 'global sales meetings', international and national meetings, and congresses. It is likely that full international travel will take a while to get back on its feet and costs are likely to remain relatively higher for some time to come.

\section{Face-to-face meetings}

These are key to many businesses, but with demonstrably effective alternatives, will distance meetings become more of a norm? Undoubtedly, there will be a surge in faceto-face meetings when it is safe to undertake these, but longer term trends will be interesting given the alternatives available.

\section{Adoption of technology}

Imagine this crisis and a lockdown without modern communications technology. Online connectivity has made a lockdown possible with millions able to perform work tasks to a useful standard while confined at home.

\section{Contact with customers}

Undoubtedly, this will change in light of lessons and new techniques finessed during this period.

\section{Sales activity and sales staff}

Again, learnings over this period will allow companies to review the best strategies and structures to enable recovery and future growth. Consideration will also need to be given to safely accessing dental premises and assessing the value that could be gained from face-to-face, rather than virtual, contact, or vice versa.

\section{Meetings and exhibitions}

Historically, these are a popular backbone of the industry and profession. Going forwards, careful consideration will need to be given to 'mass' gatherings and government rules adhered to. The industry has a moral responsibility to do what is safe for its customers and staff. With the two largest venues used by the industry for exhibitions currently accommodating Nightingale hospitals, it is difficult to see these being used for other purposes for some time yet. However, dentistry is a hands-on occupation and, traditionally, exhibitions have presented a tremendous opportunity to place product in the hands of a potential user. A time should come when exhibitions are again popular and safe places, and organisers can only plan for that future, based on best advice from the authorities and venues.

\section{CPD provision}

Will there be a further and significant drive to virtual CPD provision?

\section{Terms of trade}

Will companies be looking at different credit terms and ways of trading, with lessons learned from business closures and lockdowns?

\section{Reverse globalisation}

Will individual nations become more introverted, and will economies and governments look to more domestic manufacture of 'strategic' goods and provision of services to ensure greater national resilience against future events?

\section{The new normal}

In summary, the dental industry has a critical role alongside dental professionals to play in the return to greater levels of dental treatment. The 'new' normal will probably not look like the 'old' normal for a long time, if ever again. Things will change, but not inevitably for the worse in the longer term.

The key to getting dental activity back on track lies in:

- Reducing infection risks, to allow the;

- Resumption of dental provision, which will require the adoption of;

- New approaches, products, technologies and treatments, and inevitably;

- Changes to the structure and delivery of NHS/private dentistry.

\section{References}

1. Public Health England. Guidance: COVID-19 personal protective equipment (PPE). Available at https://www.gov. uk/government/publications/wuhan-novel-coronavirusinfection-prevention-and-control/covid-19-personalprotective-equipment-ppe (accessed April 2020). 